\title{
Transmitting and Gathering Streaming Data in Wireless Multimedia Sensor Networks Within Expected Network Lifetime
}

Lei Shu • Yan Zhang • Zhangbing Zhou •

Manfred Hauswirth • Zhiwen Yu • Gearoid Hynes

Published online: 5 August 2008

(C) Springer Science + Business Media, LLC 2008

Erratum to : Mobile Netw Appl

DOI 10.1007/s11036-008-0088-7

In Section 7.3.3, Step 2, sentences 2-3 should read as follows:

For example, in Fig. 7 node a and c have the same bearing angle, then compare the distance between neighbor node and source node aT and cT. Since cT is shorter than $\mathrm{aT}$, the node $\mathrm{c}$ is chosen as the next-hop node in this case.

The online version of the original article can be found at http://dx.doi. org/10.1007/s11036-008-0088-7.

L. Shu $(\bowtie) \cdot$ Z. Zhou $\cdot$ M. Hauswirth $\cdot$ G. Hynes

Digital Enterprise Research Institute,

National University of Ireland, Galway,

Galway, Ireland

e-mail: lei.shu@deri.org

Z. Zhou

e-mail: zhangbing.zhou@deri.org

M. Hauswirth

e-mail: manfred.hauswirth@deri.org

G. Hynes

e-mail: gearoid.hynes@deri.org

Y. Zhang

Simula Research Laboratory,

Oslo, Norway

e-mail: yanzhang@ieee.org

Z. Yu

Academic Center for Computing and Media Studies,

Kyoto University,

Kyoto, Japan

e-mail: yu@ccm.media.kyoto-u.ac.jp 